\title{
Probing radiative neutrino mass models with dilepton events at the LHC
}

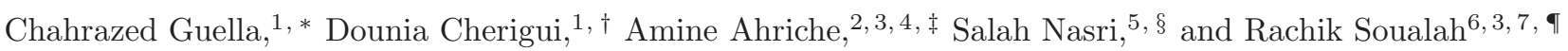 \\ ${ }^{1}$ Faculté de Physique, département de Génie Physique, \\ USTO-MB, BP1505 El M'Naouar, Oran, Algérie. \\ ${ }^{2}$ Laboratory of Mathematical and Sub-Atomic Physics (LPMPS), \\ University of Constantine I, DZ-25000 Constantine, Algeria \\ ${ }^{3}$ The Abdus Salam International Centre for Theoretical Physics, Strada Costiera 11, I-34014, Trieste, Italy. \\ ${ }^{4}$ Department of Physics and Center for Theoretical Sciences, \\ National Taiwan University, Taipei 106, Taiwan. \\ ${ }^{5}$ Department of Physics, United Arab Emirates University, Al-Ain, UAE. \\ ${ }^{6}$ Department of Applied Physics and Astronomy, \\ University of Sharjah, P. O. Box 27272 Sharjah, UAE. \\ ${ }^{7}$ INFN, Sezione di Trieste, Gruppo collegato di Udine, Italy.
}

\begin{abstract}
In this work, we investigate the possibility of probing a class of neutrino mass models at the LHC proton-proton collisions with 8 and $14 \mathrm{TeV}$ energies. The existence of lepton flavor violating interactions for a singlet charged scalar, $S^{ \pm}$, that couples to the leptons could induce many processes such as $p p \rightarrow \ell_{\alpha}^{ \pm} \ell_{\beta}^{\mp}+\not \mathbb{t}$. Using the processes with $\ell_{\alpha} \ell_{\beta}=e e, e \mu, \mu \mu$, we found that an inclusive cut on the $M_{T 2}$ event variable is vital in our analysis and leads to an effective suppression of the large Standard Model background. Our results show possible detectability of the charged scalars effect, especially at the $\sqrt{s}=14 \mathrm{TeV}$.
\end{abstract}

PACS: 12.60.-i, 12.60.Jv, 14.80.Cp, 12.15.-y.

Keywords: charged scalar, missing energy, significance.

\section{INTRODUCTION}

The Standard Model (SM) has been very successful in describing nature at the weak scale and it is in very good agreement with most of the experimental results. However depending on some of astrophysical and cosmological observations, many questions remain unanswered such as the nature of the nonbaryonic matter (dark matter), neutrino masses and their mixing, hierarchy problem, and matter-antimatter asymmetry of the Universe. Therefore, these limitations lead to the need to introduce additional components to the SM and go beyond its actual framework. There is no unique way to introduce neutrino masses in the SM. One of the attractive mechanisms that can explain the smallness of neutrino masses is the radiative mass generation mechanism. In this way, neutrinos are massless at the tree level and get a nonzero mass at the loop level which can be naturally small due to the extra loop suppression factors. This can be implemented at one loop [1], two loops [2], or three loops [3, 4], ${ }^{1}$ which can be achieved by extending the SM with new interactions involving additional scalar singlets and/or

*Electronic address: chahra.guella@gmail.com

${ }^{\dagger}$ Electronic address: dounia.ch13@gmail.com

¥Electronic address: aahriche@ictp.it

$\S$ Electronic address: snasri@uaeu.ac.ae

『 Electronic address: rsoualah@cern.ch

1 The Krauss-Nasri-Trodden model [4] has been generalized where the singlets $\mathcal{Z}_{2}$ odd singlet charged $S_{2}$, and the RH Majorana neutrinos $N_{i}$ are promoted to triplets [5], quintuplet [6], septuplets [7], and in a scale-invariant framework [8]. These models 
doublets.

In Ref. [9], the SM was extended with two electrically charged singlet fields under $S U(2)_{L}$ scalars and three right-handed $(\mathrm{RH})$ neutrinos, $N_{i}$ where a $Z_{2}$ symmetry was imposed to forbid the Dirac neutrino mass terms at tree level [4]. Once the electroweak symmetry is broken, neutrino masses are generated at three loops, and the lightest RH neutrino, $N_{1}$, could be a candidate for dark matter (DM). Some generalizations of this model have been proposed where one of the two singlet charged scalars and $N_{i}$ are replaced by $S U(2)_{L}$ triplet in Ref. [5] or by $S U(2)_{L}$ quintuplet fields [6]. The scalar spectrum of this model has a pair of charged scalar particles which can be produced at colliders via their couplings to photons and the SM higgs boson. The signatures of charged scalars at LHC and the future high-energy colliders have been studied by many authors (see, for example Refs. 12 [15]).

The main goal of our work is to present the production mechanism of the singlet charged scalar. Moreover, we

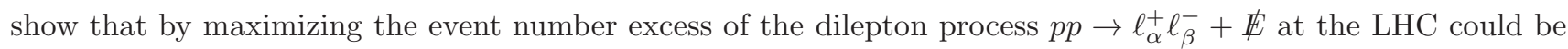
a direct probe of the charged scalar. We will confront the model parameter space with the neutrino oscillation data and the recent experimental bounds on the lepton flavor violating (LFV) processes, such as the upper limit on the $\mu \rightarrow e \gamma$ provided by the MEG Collaboration [16]. Furthermore, we impose appropriate kinematic cuts on the outgoing leptons to optimize the signal in our model over the SM background at the LHC Run I and Run II energies.

The rest of the paper is structured as follows. In Sec II , we present the class of models for neutrino mass where we describe the decay and production of the charged scalar at the LHC for c.m. (CM) energies $\sqrt{s}=8 \mathrm{TeV}$ and $\sqrt{s}=14 \mathrm{TeV}$. Section III is devoted to the study of signatures of the charged scalars at the LHC and the significance of the signal as a function of the expected luminosity. Finally, we give our conclusion in Sec. IV.

\section{CHARGED SCALARS IN RADIATIVE NEUTRINO MASS MODELS}

To generate neutrino mass at the loop level, the SM is extended by extra scalars and fermions, among which is a charged scalar, $S^{ \pm}$, that transforms under the gauge group $S U(3) \times S U(2)_{L} \times U(1)_{Y}$ as $S^{+} \sim(1,1,2)$ with the following interactions in the Lagrangian

$$
\begin{aligned}
\mathcal{L} & \supset\left\{f_{\alpha \beta} \overline{L_{\alpha}^{c}} L_{\beta} S^{+}+\text {H.c. }\right\}-M_{S}^{2} S^{+} S^{-}-V\left(H, S, \phi_{i}\right), \\
V\left(H, S, \phi_{i}\right) & \supset \lambda_{H S}|H|^{2}|S|^{2}
\end{aligned}
$$

where $L_{\alpha}=\left(\nu_{\alpha L}, \ell_{\alpha L}\right)^{T}, \ell_{\alpha R}$ is the charged lepton singlet, $f_{\alpha \beta}$ are the Yukawa couplings which are antisymmetric in the generation indices $\alpha$ and $\beta$, and $c$ denotes the charge conjugation operation. Here, $H$ represents the SM Higgs field doublet, $V\left(H, S, \phi_{i}\right)$ is the scalar potential and $\phi_{i}$ could be any additional scalar representation(s) that is (are) required to generate the neutrino mass at loop level.

In such a model, the parameter space must accommodate neutrino masses and mixing and at the same time satisfy the LFV constraints. Also, the charged scalar fields contribute to the Higgs decay channel $h \rightarrow \gamma \gamma$ and might lead to an enhancement with respect to the SM, whereas $h \rightarrow \gamma Z$ is reduced. Furthermore, a strong first-order electroweak phase transition can be achieved through the coupling of the new scalar degrees of freedom (i.e., $S$ and $\phi_{i}$ ) around the electroweak scale to the SM Higgs without being in conflict with the recent Higgs mass measurements provided by the ATLAS [17] and CM [18] collaborations. In some models, when a global symmetry $\mathcal{Z}_{2}$ is imposed, there exists a neutral Majorana particle that can be a DM candidate. The collider phenomenology of such kind of models is very rich and, in principle, can be probed through various signals.

The new interactions in Eq. (11) induce LFV effects via processes such as $\mu \rightarrow e \gamma$ and $\tau \rightarrow \mu \gamma$ and a new

phenomenology have been investigated in Refs. [9], [10] and [11]. 
contribution to the muon's anomalous magnetic moment:

$$
\begin{gathered}
B r(\mu \rightarrow e \gamma) \simeq \frac{\alpha v^{4}}{384 \pi} \frac{\left|f_{\tau e}^{*} f_{\mu \tau}\right|^{2}}{M_{S}^{4}}, \\
B r(\tau \rightarrow \mu \gamma) \simeq \frac{\alpha v^{4}}{384 \pi} \frac{\left|f_{\tau e}^{*} f_{\mu e}\right|^{2}}{M_{S}^{4}}, \\
\delta a_{\mu} \sim \frac{m_{\mu}^{2}}{96 \pi^{2}} \frac{\left|f_{e \mu}\right|^{2}+\left|f_{\mu \tau}\right|^{2}}{M_{S}^{2}},
\end{gathered}
$$

where $\alpha=e^{2} / 4 \pi$ is the fine structure constant, and $v=246 \mathrm{GeV}$ is the vacuum expectation value of the neutral component in the SM scalar doublet. The LFV branching ratios should not exceed the experimental upper bounds $\mathcal{B}(\mu \rightarrow e+\gamma)<5.7 \times 10^{-13}[16]$ and $\mathcal{B}(\tau \rightarrow \mu+\gamma)<4.8 \times 10^{-8}$ [19]. According to the LFV experimental constraints, the couplings $f$ must scale like $f \lesssim \varsigma M_{S}$, with $\varsigma$ as a dimensionful constant that depends on each bound. This means that the couplings $f$ are suppressed for low charged scalar mass values.

Here, we shall discuss the most striking features of the production mechanism and decay modes of the singlet charged scalars that can be manifested at the Large Hadron Collider. This extends our earlier studies [10] at the future $e^{-} e^{+}$colliders such as the International Linear Collider by including more accurate signatures for any possible charged scalars production ${ }^{2}$. In our analysis, we take $M_{S}$ to be within the range $[100 \mathrm{GeV}-3 \mathrm{TeV}]$ and select the parameter space to be in agreement with the LFV bounds [16].

In proton-proton collisions, the charged scalars $S^{ \pm}$can be produced in pairs through the processes:

$$
q \bar{q} \rightarrow \gamma / Z / h \rightarrow S^{+} S^{-}, g g \rightarrow h \rightarrow S^{+} S^{-},
$$

which are depicted in Fig. 1 However, the contribution of the quark-antiquark Higgs-mediated annihilation process is highly suppressed by the small Yukawa coupling of the light quarks. Thus, the production is expected to be dominated by the Drell-Yan process with the high-energy partonic cross section given by ${ }^{3}$

$$
\sigma^{(D Y)}(\hat{s})=\frac{\pi \alpha^{2}}{9 \hat{s}} \beta^{3}\left[e_{q}^{2}+\left(\frac{2 e_{q} g_{V_{q}}}{\cos ^{2} \theta_{\omega}}\right) \frac{\hat{s}}{\left(\hat{s}-M_{Z}^{2}\right)}+\left(\frac{g_{V_{q}}^{2}+g_{A_{q}}^{2}}{\cos ^{4} \theta_{\omega}}\right) \frac{\hat{s}^{2}}{\left(\hat{s}-M_{Z}^{2}\right)^{2}}\right],
$$

where $\hat{s}$ is the center-of-mass energy square of the parton system, $\beta=\sqrt{1-4 M_{S}^{2} / \hat{s}^{2}}, e_{q}$ is the electric charge of the quark, $\theta_{\omega}$ is the weak mixing angle, and $g_{V}$ and the $g_{A}$ are the SM vector and axial neutral current couplings.
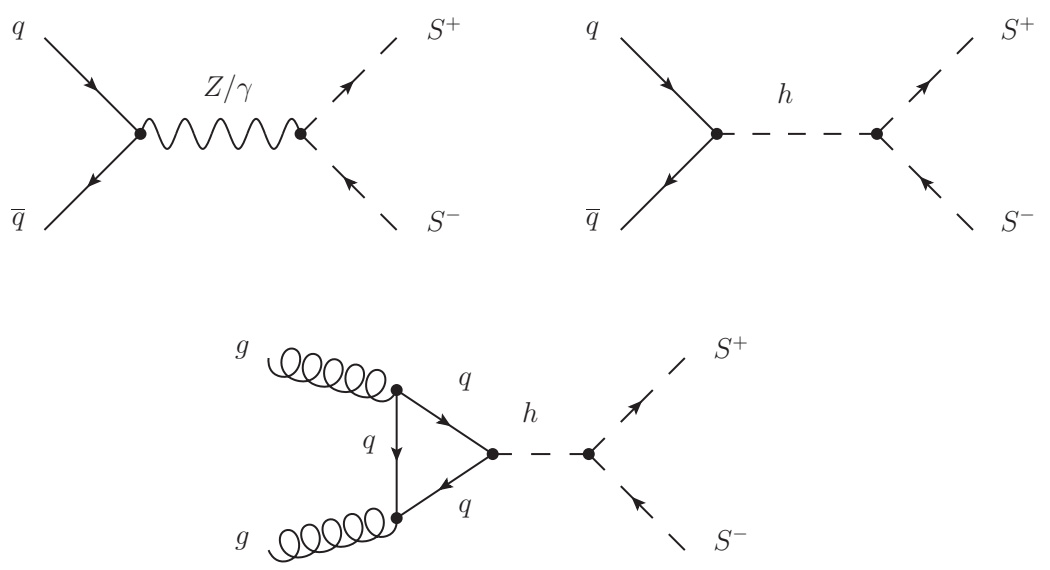

FIG. 1: The Feynman diagrams of the charged scalar $S^{ \pm}$pair production via the gluon-gluon fusion and quark-antiquark annihilation at proton-proton collisions.

\footnotetext{
2 A similar study has been performed in Ref. [20].

3 Since we are considering the charged scalar masses much heavier than $M_{Z}$, one can safely neglect the Feynman diagram with the $\mathrm{Z}$ gauge boson propagator.
} 
For a random scan of the model parameter $\left(f_{\alpha \beta}\right.$ and $\left.M_{S}\right)$, taking into account the LFV constraints and the bounds on the Higgs decay $h \rightarrow \gamma \gamma$, we show in Fig. 2 left, the cross section of the singlet charged scalars pair production as a function of the charged scalar mass $\left(M_{S}\right)$ at both $\sqrt{s}=8$ and $14 \mathrm{TeV}$, where the couplings $\lambda_{H S}$ are taken of order $\mathcal{O}(1)$. To probe how dominant the DY contribution is, we show in Fig. 2 right, the ratio $\left[\sigma^{(F u l l)}(s)-\sigma^{(D Y)}(s)\right] / \sigma^{(D Y)}(s)$ that characterizes the presence of the Higgs-mediated Feynman diagrams, where $\sigma^{(F u l l)}(s) \equiv \sigma\left(p p \rightarrow S^{+} S^{-}\right)$including the Higgs exchange diagrams.
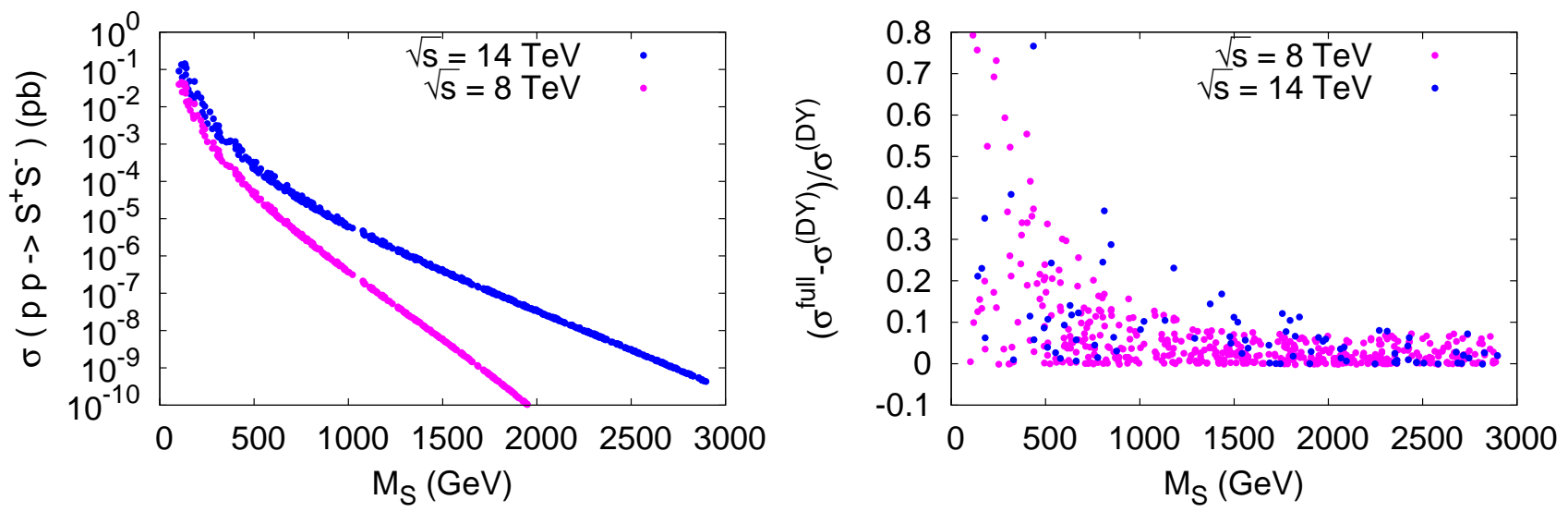

FIG. 2: Left: the production cross section (in $\mathrm{fb}$ units) of $S^{+} S^{-}$pair production at $\sqrt{s}=8 \mathrm{TeV}$ (in magenta) and $14 \mathrm{TeV}$ (in blue) vs the charged scalar mass $M_{S}$. The dashed black lines represent the DY values. Right: the ratio $\left[\sigma^{\text {full }}(s)-\sigma^{(D Y)}(s)\right] / \sigma^{(D Y)}(s)$ vs the charged scalar mass $\left(M_{S}\right)$. The considered $M_{S}$ values are in agreement with different experimental constraints such as LFV and the Higgs decay $h \rightarrow \gamma \gamma$.

One notices that the production cross section depends very strongly on the c.m. energy where it is more than 2 orders of magnitude larger for $\sqrt{s}=14 \mathrm{TeV}$ than at the $8 \mathrm{TeV}$ c.m. energy. From Fig. 2, right, we see that the DY contribution may not be the dominant one, especially for charged scalars lighter than $500 \mathrm{GeV}$.

After being produced, the charged scalar decays into a light neutrino and a charged lepton with the partial decay rate as

$$
\Gamma\left(S^{ \pm} \rightarrow \nu_{\alpha} \ell_{\beta}^{ \pm}\right)=\frac{\left|f_{\alpha \beta}\right|^{2}}{4 \pi} M_{S}\left(1-\frac{m_{\ell_{\beta}}^{2}}{M_{S}^{2}}\right)^{3 / 2} \quad ; \quad \alpha \neq \beta .
$$

Because of the fact that the $f_{\alpha \beta}$ couplings are antisymmetric, there are six decay channels in this class of models, whereas, experimentally, one can observe only three distinct signals since neutrinos are indistinguishable, i.e., charged lepton and missing energy $\left[\operatorname{Br}\left(S^{ \pm} \rightarrow \ell_{\alpha}^{ \pm}+\not{E}\right)=\sum_{\beta \neq \alpha} \operatorname{Br}\left(S^{ \pm} \rightarrow \ell_{\alpha}^{ \pm} \nu_{\beta}\right)\right]$.

For the same benchmark points used in Fig. 2, we show in Fig. 3 the charged scalar total decay width (left) and the obtained different branching ratios $\operatorname{Br}\left(S^{-} \rightarrow \ell_{\alpha}^{-}+\not\right.$ ) $)$ (right) as a function of $M_{S}$.

Furthermore, as can be seen from Fig. 3, the total decay width of the charged scalar is increasing for masses, and the branching ratios are comparable for some benchmark points. In Sec. III, we choose a benchmark point and investigate in detail the possibility of having a significant excess in the process $S^{ \pm} \rightarrow \ell_{\alpha}^{ \pm}+\not \mathbb{E}$. Such excess will be a direct proof of the effect of the charged scalars.

\section{SIGNATURE OF THE CHARGED SCALAR AT THE LHC}

In this section, we discuss one of the important tests of this class of neutrino mass models (10) which can be explored at the $\mathrm{LHC} \mathrm{TeV}$ energies. Hereafter, we consider one benchmark with the following parameters values

$$
\left\{\begin{array}{l}
f_{e \mu}=-(4.97+i 1.41) \times 10^{-2}, \quad f_{e \tau}=0.106+i 0.0859 \\
f_{\mu \tau}=(3.04-i 4.72) \times 10^{-6}, \quad M_{S}=914.2 \mathrm{GeV}
\end{array}\right.
$$



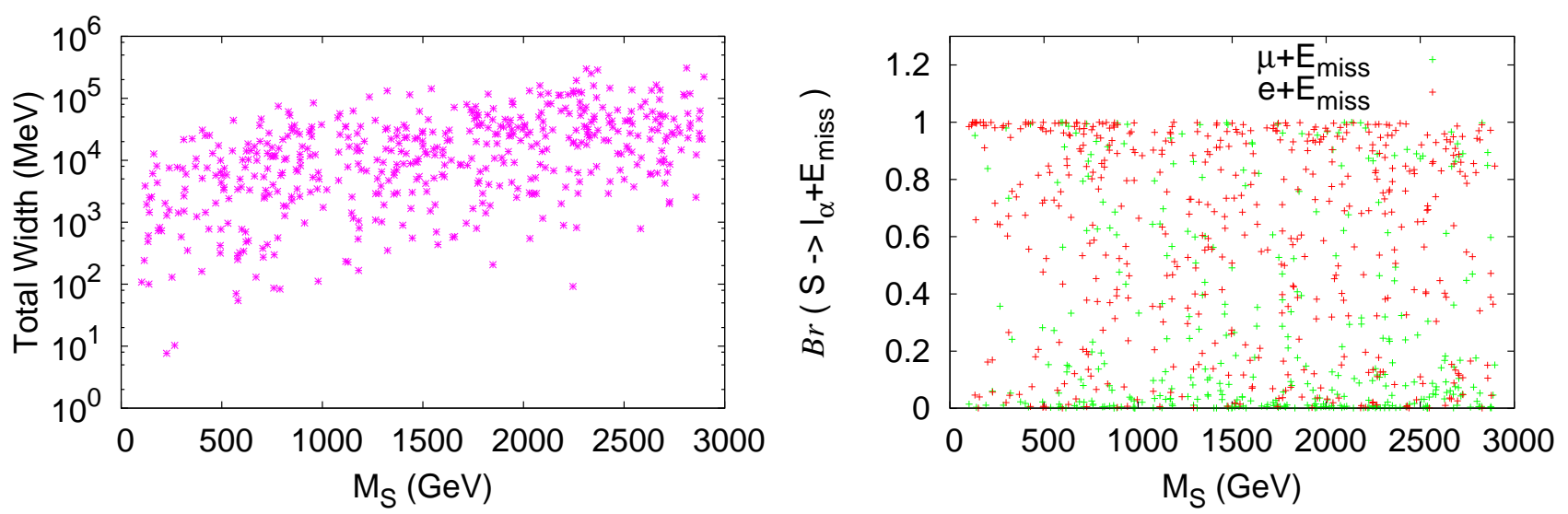

FIG. 3: The charged scalar total decay width (left) and its different branching ratios (right) vs $M_{S}$. The considered values of the charged scalar mass and the Yukawa couplings $f_{\alpha \beta}$ are in agreement with the experimental constraints mentioned above.

These considered parameters values are in agreement with LFV bounds and could be part of a benchmark point in such a model in which the neutrino oscillation data and DM relic density can be easily addressed. The signature of a charged scalar at the LHC will be identified through the detection of two opposite-sign charged leptons plus missing energy corresponding to the SM neutrinos. Therefore, the final state consists of requiring dileptons plus missing energy which will define the event topology of the signal,

$$
p p \rightarrow \ell_{\alpha}^{ \pm} \ell_{\beta}^{\mp}+\not E,
$$

where $\ell_{\alpha}^{ \pm} \ell_{\beta}^{\mp}$ can be $e^{+} e^{-}, e^{-} \mu^{+}$, or $\mu^{-} \mu^{+}$, except the $\tau$ leptons which are very difficult to identify experimentally. The missing energy $\mathbb{E}$ contribution corresponds to any of the two neutrino final states $\left\{\nu_{\alpha} \bar{\nu}_{\beta}\right\}$ with $\ell_{\alpha} \ell_{\beta}=e, \mu, \tau$ which also might include the background events produced from the leptonic decay of $W^{ \pm}$gauge bosons.

Searches for a typical signal including new charged scalars $S^{ \pm}$through kinematic distributions in events with dilepton final states have not yet found yet any significant deviation from the SM as has been illustrated by ATLAS [21] and CMS [22] collaborations. The dominant source of background is defined as being any process where $W W, Z Z$, or $Z \gamma$ are intermediate states:

$$
\begin{aligned}
& p p \rightarrow W^{+} W^{-} \rightarrow \ell_{\alpha}^{+} \ell_{\beta}^{-} \nu_{\alpha} \bar{\nu}_{\beta}, \\
& p p \rightarrow Z Z(\gamma Z) \rightarrow \ell_{\alpha}^{+} \ell_{\alpha}^{-} \nu_{\beta} \bar{\nu}_{\beta} .
\end{aligned}
$$

According to the interactions in Eq. (10), the same final state can be achieved through intermediate states that include a single or a pair of charged scalars. Thereby, in this work, we are looking for any deviation from the SM where the effective cross section in question is the difference between the cross section estimated according to Eq. (1) and the one estimated within the SM. Thus, when finding the different cuts, we consider the values of kinematic variables for which the effective differential cross section is strictly positive.

In this work, the model files were produced using the LanHEP program 23] for the Feynman rules generation in momentum representation. Then the event generation and simulation for the corresponding cross sections of both signal and background processes at various c.m. energies were obtained by using CalcHEP [24].

To probe the effect of lepton universality violating processes due to the interactions given in Eq. (1), we show in Fig. 团 the cross section $\sigma\left(p p \rightarrow \ell_{\alpha}^{ \pm} \ell_{\beta}^{\mp}+\mathbb{E}\right)$ that corresponds to the three cases $\left(\ell_{\alpha} \ell_{\beta}=e e, e \mu, \mu \mu\right)$ vs the c.m. energies $\sqrt{s}=[7 \mathrm{TeV}-100 \mathrm{TeV}]$.

As can be seen, for the SM case, the two processes $p p \rightarrow e^{+} e^{-}+\not \mathbb{E}$ and $p p \rightarrow \mu^{+} \mu^{-}+\not \mathbb{E}$ have the same cross section values, while in our model it shows an increasing difference with respect to the c.m. energy values. This can be understood due to the fact that the charged scalar couplings to the leptons are not universal. Therefore, it is possible to test these interactions when more data are accumulated at the LHC Run II. 


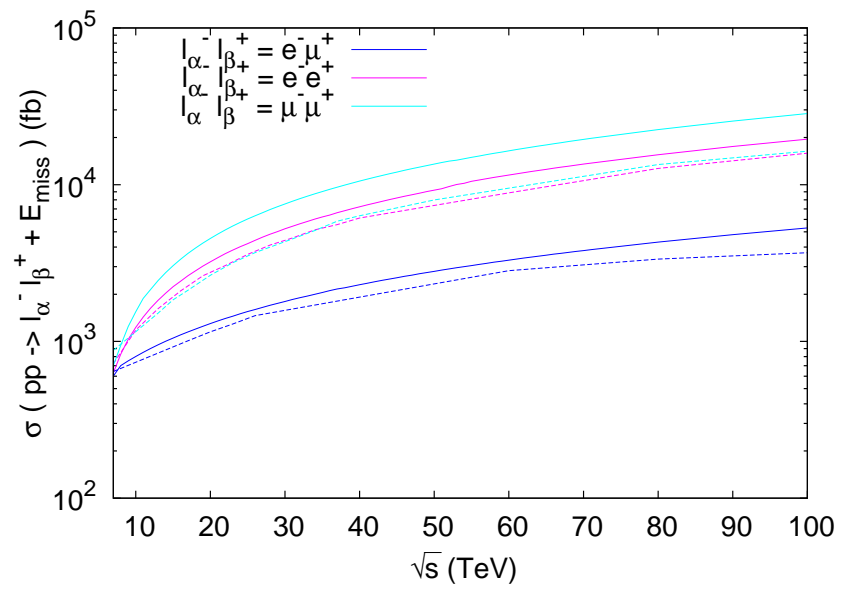

FIG. 4: The cross section (in fb units) of different contributions (solid lines) of the missing energy for each process as a function of the center of mass (in $\mathrm{TeV}$ ). Here, the dashed lines represent the SM values.

\section{A. Signal and background}

The criteria used to reject the background are based on the full kinematic study of the events. For that, we have examined the kinematic distributions of the signal and background to define a convenient set of cuts that can provide a good discrimination against the reducible background.

To optimize the signal significance, the event selection is performed in two steps: an initial preselection and final selection. In the preselection we use an accurate cut on the $M_{T 2}$ event variable 25, 26] to separate as much as possible the signal from the SM background without a large reduction in the signal efficiency. This is applied by imposing an inclusive cut on $M_{T 2}$ defined as

$$
M_{T 2}=\min _{\vec{p}_{T}^{(\nu \alpha)}+\vec{p}_{T}^{\left(\nu_{\beta}\right)}=\vec{p}_{T}^{m i s s}}\left[\max \left(M_{T}^{(\alpha)}+M_{T}^{(\beta)}\right)\right]
$$

with

$$
M_{T}^{(\alpha)}=m_{\ell_{\alpha}}^{2}+2\left(E_{T}^{\ell_{\alpha}} E_{T}^{\nu_{\alpha}}-\vec{p}_{T}^{\ell_{\alpha}} \cdot \vec{p}_{T}^{\nu_{\alpha}}\right)
$$

where $E_{T}^{\ell_{\alpha}}\left(E_{T}^{\nu_{\alpha}}\right)$ and $\vec{p}_{T}^{\ell_{\alpha}}\left(\vec{p}_{T}^{\nu_{\alpha}}\right)$ are the energy and transverse momentum energy of the charged lepton (neutrino). The $M_{T 2}$ variable, which is the transverse momentum imbalance in an event, is used to bound the masses and to reflect the masses of the pair-produced particles. In the limit of massless missing energy particles, the $M_{T 2}$ variable can be written as [27]

$$
M_{T 2}^{2}=2 p_{T}^{\ell_{\alpha}} p_{T}^{\ell_{\beta}}\left(1+\cos \theta_{\alpha \beta}\right),
$$

and $\theta_{\alpha \beta} \equiv\left(\ell_{\alpha}, \ell_{\beta}\right)$ is the angle between the charged leptons. As a result, in pair-production events with two leptons and missing energy, the $M_{T 2}$ distribution has an upper limit at the mass of the mother particle. Therefore, the background events of the charged leptons and neutrinos coming from the $W^{+} W^{-}$source can be eliminated by imposing an inclusive- $M_{T 2}$ cut expressed as

$$
M_{T 2}>M_{W} .
$$

After imposing the condition (15), we study different distributions of the considered processes at both 8 and $14 \mathrm{TeV}$ c.m. energies, and then deduce the relevant kinematic cuts as summarized in Table [I

Note that some cuts are set as upper bounds on charged leptons energies and transverse momenta. These are required to avoid a negative effective deferential cross section. When comparing the charged leptons cut values of the two processes $p p \rightarrow e^{+} e^{-}+\not \mathbb{E}$ and $p p \rightarrow \mu^{+} \mu^{-}+\not \mathbb{E}$, one remarks that an $e-\mu$ asymmetry exists, which gets larger at higher c.m. energies. This can be understood due to nonuniversal couplings of the charged scalars to a lepton in Eq. (11) as it has been stated in the previous section. 


\begin{tabular}{|c|c|c|}
\hline Process & Cuts@8 TeV & Cuts@14 TeV \\
\hline$p p \rightarrow e^{-} \mu^{+}+\not t$ & $\begin{array}{cc}80<p_{T}^{e^{-}}<250 & 80<p_{T}^{\mu^{+}}<270 \\
-1.560<\eta_{e^{-}}<2.99 & -1.92<\eta_{\mu^{+}}<3 \\
\end{array}$ & $\begin{array}{cc}p_{T}^{e^{-}}>180 & p_{T}^{\mu^{+}}>170 \\
1.1<\eta_{e^{-}}<2.89 & 1.2<\eta_{\mu^{+}}<3.02 \\
\end{array}$ \\
\hline$p p \rightarrow e^{-} e^{+}+\not t$ & $\begin{array}{c}25<p_{T}^{l}<120 \\
-2.09<\eta_{l}<2.89 \\
\end{array}$ & $\begin{array}{c}30<p_{T}^{l}<80 \\
-2.8<\eta_{l}<2.95 \\
\end{array}$ \\
\hline$p p \rightarrow \mu^{-} \mu^{+}+\not \notin$ & $\begin{array}{c}30<p_{T}^{l}<155 \\
-2.38<\eta_{l}<2.1\end{array}$ & $\begin{array}{c}25<p_{T}^{l}<40 \\
-0.13<\eta_{l}<3\end{array}$ \\
\hline
\end{tabular}

TABLE I: The considered cuts for the three final states at $\sqrt{s}=8$ and 14 TeV center-of-mass energy. The $p_{T}^{\ell}$ and $\eta_{\ell}$ are, respectively, the transverse momentum and pseudorapidity of the charged lepton $(e, \mu)$.

\section{B. Numerical results}

The event yields which pass the kinematic cuts under the conditions described in Table \ will be used to evaluate the physics significance of the signal. We followed a detailed kinematic variables scan where a tightened cut selection is taken into account in order to optimize the separation of the signal to the background ratio. For each signal final state, the signal significance is defined as

$$
S=\frac{N_{e x}}{\sqrt{N_{e x}+N_{B}}},
$$

where $N_{e x}$ is the excess events number of the considered signal and $N_{B}$ is the number of events of the background contributions that can mimic the signal. More explicitly, this excess can be expressed as

$$
N_{e x}=N_{M}-N_{B}=L \times\left(\sigma^{M}-\sigma^{B}\right),
$$

where $N_{M}$ is the expected events number due to all the new model interactions including those of the SM, $L$ is the integrated luminosity (known also as $\int \mathcal{L} d t$ ), and $\sigma^{M}\left(\sigma^{B}\right)$ is the total expected (background) cross section. In summary, Table II presents the signal and background cross sections at 8 and 14 TeV for the three different signatures after passing the selection cuts given earlier. The corresponding significance depending on the available

\begin{tabular}{|c|c|c|c|c|c|c|c|c|}
\hline \multirow[b]{2}{*}{ Process } & \multicolumn{4}{|c|}{$\sqrt{s}=8 \mathrm{TeV}$} & \multicolumn{4}{|c|}{$\sqrt{s}=14 \mathrm{TeV}$} \\
\hline & $\sigma^{M}(f b)$ & $\sigma^{B}(f b)$ & $\left(\sigma^{M}-\sigma^{B}\right) / \sigma^{B}$ & $S_{20}$ & $\sigma^{M}(f b)$ & $\sigma^{B}(f b)$ & $\left(\sigma^{M}-\sigma^{B}\right) / \sigma^{B}$ & $S_{100}$ \\
\hline$p p \rightarrow e^{-} \mu^{+}+\not E$ & 13.03 & 11.98 & 0.0876 & 1.301 & 1.253 & 0.459 & 1.7 & 7.093 \\
\hline$p p \rightarrow e^{-} e^{+}+\not \mathbb{E}$ & 62.74 & 59.72 & 0.0506 & 1.7051 & 44.45 & 38.65 & 0.150 & 8.699 \\
\hline$p p \rightarrow \mu^{-} \mu^{+}+\not E$ & 81.691 & 77.49 & 0.0542 & 2.0786 & 65.27 & 56.86 & 0.148 & 10.409 \\
\hline
\end{tabular}
(and the expected with $14 \mathrm{TeV}$ ) luminosity is then extracted and written in the last column of each table.

TABLE II: The cross section of the total expected signals $\left(\sigma^{M}\right)$ and the corresponding background $\left(\sigma^{B}\right)$ are used to estimate the significance $S_{20}\left(S_{100}\right)$ at $8 \mathrm{TeV}(14 \mathrm{TeV})$ with the recorded integrated luminosity

$$
L=20 \mathrm{fb}^{-1}\left(L=100 \mathrm{fb}^{-1}\right) \text {. }
$$

The numerical results show that the signal significance varies in the range of $[1.30-2.07]$ at $\sqrt{s}=8$ TeV with an optimum value up to 10.41 at $\sqrt{s}=14 \mathrm{TeV}$. It should be pointed out that the final states with the charged leptons $\mu^{-} \mu^{+}$are significantly larger than $e^{-} e^{+}$, and as a consequence, it is the most favorable channel to tackle the singlet charged pair production at both collider energies. A convenient representation of these results is also illustrated in Fig. 5 where the signal significance is plotted against the available and expected luminosity regions.

Having shown the consistency of all the possible signal signatures at both energies, it is justified to explore furthermore the phase space to determine their behavior based on one of the main model parameters which is the mass of the charged scalar. In Fig. 6, we show the dependence of the significance vs $M_{S}$ at both c.m. energies 8 and $14 \mathrm{TeV}$ with $L=20 \mathrm{fb}^{-1}$ (left) and $L=100 \mathrm{fb}^{-1}$ (right). In this figure, the branching fractions for the LFV processes $\ell_{\alpha} \rightarrow \ell_{\beta}+\gamma$ have been taken to be equal to the value computed using the parameters given in Eq. (9) and which are just below the LFV bounds. 

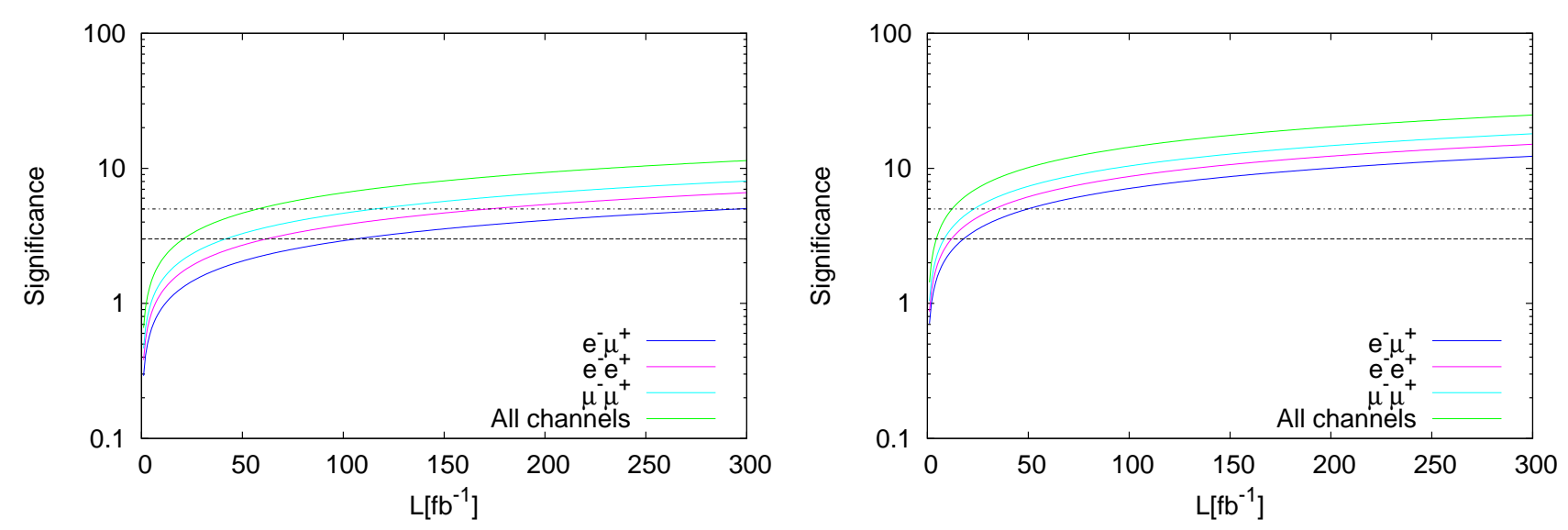

FIG. 5: The significance vs the luminosity at $\sqrt{s}=8 \mathrm{TeV}$ (left) and at $\sqrt{s}=14 \mathrm{TeV}$ (right) for the three different signatures. The two horizontal dashed lines in each panel indicate the corresponding significance values for $S=3$ and $S=5$, respectively.
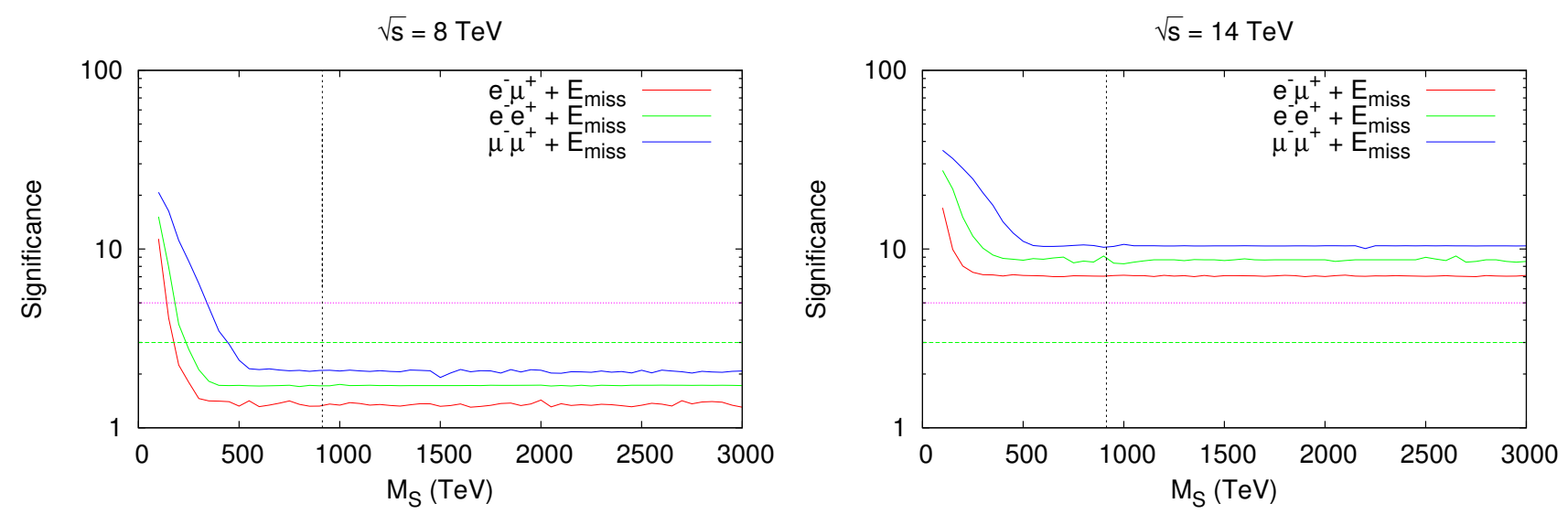

FIG. 6: The significance vs the mass of the charged scalar $M_{S}$ for each channel at $\mathrm{L}=20 \mathrm{fb}^{-1}$ (left) and at L $=100 \mathrm{fb}^{-1}$ (right). The two dashed horizontal lines indicate the significance values at $\mathrm{S}=3$ and $\mathrm{S}=5$, and the vertical one indicates the charged scalar mass given (9).

As can be seen in Fig. [6] left, the LHC Run I data can be used to exclude the charged scalars with masses below $400 \mathrm{GeV}$, whereas Fig. 6. right, shows that the signal is likely to be seen at LHC@14. Note that for benchmarks with $\operatorname{Br}\left(\ell_{\alpha} \rightarrow \ell_{\beta}+\gamma\right)$ much smaller than the current experimental bound the significance gets reduced since in this case the couplings $f_{\alpha \beta}$ are tiny and/or $M_{S}$ is very large.

\section{CONCLUSION}

In this work, we have investigated the possibility of testing the singlet charged scalars effect in a class of neutrino mass models. This dedicated search at the LHC energies can place stringent limits on the cross section times the branching ratios of the charged scalar decaying to leptons in the radiative neutrino mass models. We considered the opposite-sign dilepton final states with missing energy at the LHC proton-proton collisions with both 8 and $14 \mathrm{TeV}$ c.m. energies. An observed deviation from the SM, if seen in the future, could be a very important hint that leads us to consider the SM left-handed neutrinos as Majorana fermions. We found that these charged 
scalars can be pair produced at the LHC and decay inside the detector. To extract the contribution of the charged scalars, we analyzed the dilepton final states with missing energy $\left(p p \rightarrow \ell_{\alpha}^{ \pm} \ell_{\beta}^{\mp}+\not t\right)$, where $\ell_{\alpha} \ell_{\beta}=e e, e \mu, \mu \mu$. We concluded that an inclusive cut on the $M_{T 2}$ event variable is vital and has an effective suppression of the large SM background. We found that at the LHC@8 TeV the charged scalars effect on $p p \rightarrow \ell_{\alpha}^{ \pm} \ell_{\beta}^{\mp}+\not E$ cannot be seen with the integrated luminosity $L=20 \mathrm{fb}^{-1}$, and no significant deviations are observed. However, at the LHC@14 TeV with the expected integrated luminosity $L=100 \mathrm{fb}^{-1}$, an effect can be found in all channels. Moreover, it has been shown that the signal significance decreases rapidly with the increasing charged scalar mass. For instance, when $M_{S}=912.4 \mathrm{GeV}$, it leads to $3 \sigma$ significance of for $L=20 \mathrm{fb}^{-1}$ and more than $5 \sigma$ significance for $L=100$ $f b^{-1}$, and the favored channel would be $p p \rightarrow \mu^{+} \mu^{-}+\not t$ at both 8, $14 \mathrm{TeV}$ c.m. energies.

\section{Acknowledgments}

We would like to thank K. Cheung for his useful comments and A.B. Hammou for reading the manuscript. A. A., C. G., and D. C. would like to thank the ICTP for the warm hospitality during part of this work. A. A. is supported by the Algerian Ministry of Higher Education and Scientific Research under the CNEPRU Project No. D01720130042.

[1] A. Zee, Phys. Lett. B 161, 141 (1985); E. Ma, Phys. Rev. Lett. 81, 1171 (1998).

[2] A. Zee, Nucl. Phys. B 264, 99 (1986); K. S. Babu, Phys. Lett. B 203, 132 (1988); M. Aoki, S. Kanemura, T. Shindou, and K. Yagyu, J. High Energy Phys. 101007 (2010) 084; J. High Energy Phys. 11 (2010) 049; G. Guo, X. G. He, and G. N. Li, J. High Energy Phys. 10 (2012) 044; Y. Kajiyama, H. Okada, and K. Yagyu, Nucl. Phys. B874, 198 (2013).

[3] M. Aoki, S. Kanemura, and O. Seto, Phys. Rev. Lett. 102, 051805 (2009); M. Aoki, S. Kanemura, and O. Seto, Phys. Rev. D 80, 033007 (2009).

[4] L. M. Krauss, S. Nasri, and M. Trodden, Phys. Rev. D 67, 085002 (2003).

[5] A. Ahriche, C. S. Chen, K. L. McDonald, and S. Nasri, Phys. Rev. D 90, 015024 (2014).

[6] A. Ahriche, K. L. McDonald, and S. Nasri, J. High Energy Phys. 10 (2014) 167.

[7] A. Ahriche, K. L. McDonald, S. Nasri, and T. Toma, Phys. Lett. B 746, 430 (2015).

[8] A. Ahriche, K. L. McDonald, and S. Nasri, JHEP 1602 (2016) 038 arXiv:1508.02607 [hep-ph]].

[9] A. Ahriche, and S. Nasri, J. Cosmol. Astropart. Phys. 07 (2013) 035.

[10] A. Ahriche, S. Nasri, and R. Soualah, Phys. Rev. D 89, 095010 (2014).

[11] A. Ahriche, K. L. McDonald, and S. Nasri, Phys. Rev. D 92, 095020 (2015).

[12] P. Osland, A. Pukhov, G. M. Pruna, and M. Purmohammadi, J. High Energy Physics. 04 (2013) 040.

[13] K. Cheung and O. Seto, Phys. Rev. D 69, 113009 (2004).

[14] F. del Águila and M. Chala, J. High Energy Phys. 03 (2014) 027.

[15] E. J. Chun, and P. Sharma, Phys. Lett. B 728, 256 (2014).

[16] J. Adam et al. (MEG Collaboration), Phys. Rev. Lett. 110, 201801 (2013).

[17] G. Aad et al. (ATLAS Collaboration), Phys. Lett. B 716, 1 (2012).

[18] S. Chatrchyan et al. (CMS Collaboration), Phys. Lett. B 716, 30 (2012).

[19] K. A. Olive et al. (Particle Data Group Collaboration), Chin. Phys. C 38, 090001 (2014).

[20] S. Kanemura, T. Nabeshima, and H. Sugiyama, Phys. Rev. D 87, 015009 (2013).

[21] G. Aad et al. (ATLAS Collaboration), Eur. Phys. J. C 72, 2244 (2012).

[22] S. Chatrchyan et al. (CMS Collaboration), Eur. Phys. J. C 72, 2189 (2012).

[23] A. Semenov, Comput. Phys. Commun. 180, 431 (2009).

[24] A. Belyaev, N. D. Christensen, and A. Pukhov, Comput. Phys. Commun. 184, 1729 (2013).

[25] C. G. Lester, and D. J. Summers, Phys. Lett. B 463, 99 (1999).

[26] A. Barr, C. Lester, and P. Stephens, J. Phys. G 29, 2343 (2003).

[27] S. Chatrchyan et al. (CMS Collaboration), J. High Energy Phys. 10 (2012) 018. 